\title{
COVID-19 or Not, Breast Cancer Treatment Must Go On
}

\author{
Alexandru Blidaru* \\ "Carol Davila" University of Medicine and Pharmacy, "Prof. Dr. Al. Trestioreanu" Institute of Oncology, Bucharest, Romania \\ Romanian Society of Breast Surgery and Oncology
}

${ }^{*}$ Corresponding author: Professor Alexandru Blidaru "Carol Davila" University of Medicine and Pharmacy, Bucharest, Romania Department of Surgical Oncology, "Prof. Dr. Al. Trestioreanu" Institute of Oncology, Bucharest, Romania E-mail: alexandrublidaru@yahoo.com
When at the end of last year Professor Traian Patrascu asked me to coordinate an issue of the journal "Chirurgia" dedicated to breast cancer, I was both surprised and intrigued. For two reasons: firstly it seemed to me so little time had passed since the previous issue about this disease and perhaps there weren't many new things to say and secondly I believed that the only topic worth discussing nowadays was the disease that overshadowed anything else: COVID-19. Nevertheless I realized that he was right. More than 3 years had passed since the previous issue and there were many updates related to this subject and COVID-19 or not, the treatment of breast cancer must continue.

I am grateful to those who answered to my invitation to write an article. I was amazed by the huge number and the quality of the papers received. They were so numerous that we decided to have a supplement of the journal dedicated to breast cancer edited with the contribution of the Romanian Society of Breast Surgery and Oncology.

The COVID-19 pandemic has affected the entire planet in almost every aspect of life, including the medical system. The treatment of other diseases, other than COVID-19, has in some way become secondary, at least for the time being. This had immediate repercussions and will inevitably have a long-term impact as well. Breast cancer patients suffered consequently. Screening, diagnosis, treatments including surgery and post therapeutic follow-ups have been profoundly affected by the pandemic.

The COVID-19 pandemic is an immeasurable human tragedy. We mourn the suffering and death of so many people and many colleagues and friends and we bow to their memory.

The beginning of the pandemic was a major shock, uncertainty 
heightened and numerous scenarios were deliberated that were not confirmed by the evolution of the disease. Furthermore numerous attempts were made to cease the spread of the COVID-19 pandemic that unfortunately did not offer the expected results.

We did not experience as predicted a "first wave" followed months later by a "mild second wave", instead a less predictable pattern took place, the disease manifesting in different ways around various regions of the globe. Some states initially have chose to achieve herd immunity others implemented quarantine measures followed by partial relaxation and requarantined. Lock-down, relaxation, lock-down again. None achieved the expected results, which accentuated the feeling of confusion. Several medications proposed initially that seemed lifesaving later proved ineffective. The only worthwhile measures in combating this disease remained physical distancing, wearing masks and hand washing. We can only hope that the vaccine stops the disease, but it seems that a "third huge wave" is already here. Confronted with this pandemic, people, including medical staff, politicians, heads of state, governments, non-profit organizations, international organizations have gone through the five stages of grief denial, anger, bargaining, depression and acceptance. In many cases some remained confined in one of this stages.

The COVID-19 pandemic "first wave" did not take place concomitantly around the world, resulting in some states being able to prepare ahead of time for the coming pandemic, organizing their health system to cope with the shock produced by COVID-19 pandemic.

Many wards and hospitals were emptied of patients and in the first few weeks breast cancer cases were operated and treated only in case of surgical or oncological emergency. Very useful at that time were the guidelines of COVID-19 Breast Cancer Consortium (1). During the early stages of the pandemic mainly simple operations were performed, on cases that could not be postponed or delayed with systemic treatment. The measures taken during this period have led to an interruption of routine medical treatments for a vulnerable category of patients whose results depend on timely and high quality multidisciplinary interventions. Breast reconstructions and contralateral surgeries for symmetry and riskreducing purposes were not performed in order to reduce operating time, to lower the risk of complications and to limit the number of necessary staff in the operating room. Patients have undergone triage procedures and testing before being admitted to hospitals.

In the United States, for example, when hospitals in large cities were overflowing with COVID-19 patients and even operating rooms were transformed into intensive care units, surgical teams were detached along with patients to smaller cities where surgeries could be performed.

Each country has adopted different strategies: some have appointed certain hospitals only for COVID-19 cases, others have made "breast regional cancer hubs" to organize the management of this disease. This type of medical facility brings together multidisciplinary teams consisting of: surgeons, oncologists, plastic surgeons, imaging specialists, pathologists and radiotherapists (2)

There were also astonishing situations; according to a colleague from a neighboring country, hospitals were emptied waiting for COVID-19 patients and for a long time COVID19 patients did not come. However, after some months the country was overflowed with COVID-19 patients.

When we realized that this pandemic would not pass quickly and that we have to adapt for longtime situations we began to resume medical and surgical treatments, performing oncoplastic surgery, breast reconstruction, prophylactic surgery, always taking into consideration the endemic situation and resources available. In terms of resources many funds and personnel have been redirected to aid the diagnosis and treatment of COVID-19.

Another matter that will have a long-term impact is the fact that patients have missed screening appointments, they have not performed investigations on their own initiative, opportunistic screening, and have not visited a physician even when presenting symptoms (3). 
Moreover they did not attend the posttherapeutic follow-ups. The fear of leaving the house and going to the hospital, which most associate with a source of illness, explains to some extent this behavior. Consultation at a physician during this period, for breast cancer as well as for any other medical condition, other than COVID-19, decreased. Telemedicine tried to partially replace traditional face-to-face medicine.

Beside the diagnosis of cancer, the psychological disorders caused by the pandemic affected patients and psychological and even psychiatric support has become even more important.

As the epidemiological situation improved, hospitals were prepared to receive patients with conditions other than COVID-19, but the patients did not come to the hospital. In conclusion, delayed diagnosis is likely to have a significant impact on breast cancer mortality in the years to come.

Breast cancer patients have a clear indication for anti-COVID-19 vaccine and the occurrence of a reactive axillary lymphadenopathy after this type of vaccine must be differentiated from an oncological sign. The recommendation is that the vaccine be administered in the contralateral arm to the location of the cancer. On the other hand, the discovery of lymphadenopathy after a vaccine in a healthy person can be revealing for a diagnosis of malignancy. In such cases, in addition to imaging investigations, biopsy (with both histopathological and cytological examination) may be necessary.

Systemic treatments (endocrine, cytostatic, monoclonal antibody treatments and other molecular therapies) have never ceased during the pandemic. Classical schemes were used but "dose-dense" regimens were used sparingly. During the early stages of the pandemic, neoadjuvant treatment was performed in order to postpone surgery.

Real-time PCR tests for COVID-19 infection were preformed pre-therapeutically and at twoweek intervals during treatment.

During cytostatic treatment with Anthracyclines/Taxanes, the primary prophylactic treatment regarding medullar toxicity was extended from two to five days, thus reducing the risk of febrile neutropenia, which could easily be confused with the onset of COVID-19.

Upon initiation of the anti-COVID-19 vaccination campaign in patients undergoing chemotherapy it was recommended to get the vaccine 3-4 days after the chemotherapy session. In patients with breast cancer undergoing cytostatic treatment, anti-influenza and anti-pneumococcal vaccinations have been recommended in order to lower the complications of COVID-19 disease.

International guidelines for radiation therapy in breast cancer during the COVID-19 period have been elaborated (4). The most important recommendations were hypofractionation, omission of the boost and even omission of radiotherapy for low-risk patients.

Without regard of Covid 19, breast cancer treatment has been through a continuous evolution. The journey had ups and downs looking for the best way to treat in order to achieve both improved survival and quality of life. The trend was de-escalation of the treatment. It seemed to me that only surgery was heading in that direction, "from maximum tolerable to minimum effective treatment" as professor Umberto Veronesi recommended and that the other treatments for breast cancer did not keep this path (5). But escalation and deescalation was observed for all types of oncological treatments for breast cancer. Surgery evolved from mastectomy with axillary clearance to conservative treatment and selective axillary dissection. In recent years breast cancer surgery have become more complex and diverse: oncoplastic surgery, prophylactic mastectomy, different types of breast reconstruction. Talking with my colleagues and friends dr. Daniela Zob, dr. Liviu Stoleru, and dr. Dan Jinga I understood that systemic treatment and radiotherapy for breast cancer are in a continuous evolution and that gold-standards are fluid. It should have been obvious as gold is the most malleable and soft of all metals.

In regards to chemotherapy there has been a escalation in treatment of triple-negative and 
Her2 positive breast cancer patients. In patients with triple negative breast cancer platinum derivatives have been introduced both in neoadjuvant and adjuvant treatment. In the absence of complete histopathological response adjuvant Capecitabine was added. In Her2 neu positive neoadjuvant treatments is represented by: Trastuzmab + Pertuzumab + chemotherapy and if complete histopathological response is not achieved TD-M1 is recommended in adjuvant therapy $(6,7)$.

De-escalation of the treatment was attained in hormone positive, Her2 negative patients. Based on genomic testing (Oncotype Dx, PAM50, MammaPrint, Prosigna) the recurrence score and the benefit of chemotherapy can be established and thus selecting the appropriate treatment $(8,9)$. Using this tests lead to omission of chemotherapy in more than $50 \%$ of patients.

For radiotherapy even though the indications after mastectomy have increased there are still categories of patients in which radiotherapy can be omitted.

Omission of radiotherapy can be recommended in patients over the age of 65 or in young patients with significant comorbidities with invasive breast cancer smaller than 30 $\mathrm{mm}$, negative margins, grade $1-2$, positive estrogen receptors, Her2 negative and node negative. This patients have to be scheduled for endocrine therapy. Hypofractionation and total dose reduction is another example of deescalation of radiotherapy of the whole breast. The indication for hypofractionated radiation therapy of the whole breast extended to patients with lymph node metastasis, but longterm aesthetic results are to be assessed. Various techniques and fractionation regimens are available for partial breast irradiation. Accelerated Partial Breast Irradiation has been validated as having long-term oncological outcomes comparable to standard whole breast radiotherapy in low-risk patients (10).

All these examples confirm the dynamic nonlinear evolution of breast cancer treatment.
In the absence of appropriate treatment the lives of breast cancer patients are in danger. COVID-19 pandemic is affecting in multiple ways the mortality related to breast cancer.

The number of newly diagnosed breast cancer cases in the world has decreased not due to a reduction of incidence, but because of underdiagnose. The time span from diagnosis to initiation of treatment is an important prognostic factor. Screening programs need to be restarted and the best oncological treatment has to be offered to patients with breast cancer despite the pandemic.

COVID-19 or not, breast cancer treatment must go on.

\section{Conflict of Interest}

The authors declare no conflicts of interests.

\section{Reference}

1. Ahmed M. Optimizing breast cancer surgery during the COVID-19 pandemic. Breast Cancer. 2020;27(6):1045-1047.

2. Veronesi P. Corso G. Impact of COVID 19 pandemic on clinical and surgical breast cancer management. EClinicalMedicine. 2020 Sep; 26:100523.

3. Covid-19 Breast Cancer Patient Triage Guideliness (CPBCC, 2020), April 8, 2020; https://www.webmd.com/lung/news/20200408/ covid-19-breastcancer-patient-triage-guidelines-cpbcc-2020\#1

4. Coles CE, Aristei C, Bliss J, Boersma L, Brunt AM, Chatterjee S, et al. International Guidelines on Radiation Therapy for Breast Cancer During the COVID-19 Pandemic. Clin Oncol (R Coll Radiol). 2020; 32(5):279-281.

5. Curigliano G, Cardoso F, Costa A, Galimberti V, Goldhirsch A, Pelicci PG, et el. From the maximum tolerable to the minimum effective treatment: The Umberto Veronesi's life commitment to breat cancer care. Breast. 2017;31: 241-243.

6. A study of Pertuzumab in Addition to Chemotheraphy and Trastuzumab as Adjuvant Therapy in Participants with Human Epiderma Growth Receptor 2 *HER2)-Positive primary breast cancer (APHINITY). Clinical Trials.gov Identifier: NCT01358877.

7. Huang CS, Mano MS, Loibl S, Mamounas EP, Untch M, et al. Trastuzumab Emtansine for Residual Invasive HER2-Positive Breast cancer. N Engl J Med. 2019;380(7):617-628.

8. Sparano JA, Gray RJ, Makower DF, Pritchard KI, Albain KS, Hayes DF, et al. Adjuvant chemotherapy guided by a 21-gene expression assay in breast cancer. N Engl J Med. $2018 ; 379(2): 111-121$.

9. Cardoso F, van 't Veer L, Poncet C, Lopes Cardozo J, Delaloge S, Pierga JY, et al. MINDACT: Long-term results of the large prospective trial testing the 70-gene signature MammaPrint as guidance for adjuvant chemotherapy in breast cancer patients. Journal of Clinical Oncology. 2020; 38(15):506-506.

10. Forster T, Köhler CVK, Debus J, Hörner-Rieber J. Accelerated Partial Breast Irradiation: A new standard of care? Breast Care (Basel). 2020;15(2): 136-147. 